\title{
The association between neighbourhood characteristics and physical victimisation in men and women with mental disorders
}

Vishal Bhavsar, Jyoti Sanyal, Rashmi Patel, Hitesh Shetty, Sumithra Velupillai, Robert Stewart, Matthew Broadbent, James H. MacCabe, Jayati Das-Munshi and Louise M. Howard

\section{Background}

How neighbourhood characteristics affect the physical safety of people with mental illness is unclear.

\section{Aims}

To examine neighbourhood effects on physical victimisation towards people using mental health services.

\section{Method}

We developed and evaluated a machine-learning-derived freetext-based natural language processing (NLP) algorithm to ascertain clinical text referring to physical victimisation. This was applied to records on all patients attending National Health Service mental health services in Southeast London. Sociodemographic and clinical data, and diagnostic information on use of acute hospital care (from Hospital Episode Statistics, linked to Clinical Record Interactive Search), were collected in this group, defined as 'cases' and concurrently sampled controls. Multilevel logistic regression models estimated associations (odds ratios, ORs) between neighbourhood-level fragmentation, crime, income deprivation, and population density and physical victimisation

\section{Results}

Based on a human-rated gold standard, the NLP algorithm had a positive predictive value of 0.92 and sensitivity of 0.98 for (clinically recorded) physical victimisation. A 1 s.d. increase in neighbourhood crime was accompanied by a $7 \%$ increase in odds of physical victimisation in women and an $13 \%$ increase in men (adjusted OR (aOR) for women: 1.07, 95\% Cl 1.01-1.14, aOR for men: 1.13, 95\% Cl 1.06-1.21, $P$ for gender interaction, 0.218). Although small, adjusted associations for neighbourhood fragmentation appeared greater in magnitude for women $(\mathrm{aOR}=1.05,95 \% \mathrm{Cl} 1.01-1.11)$ than men, where this association was not statistically significant $(\mathrm{aOR}=1.00,95 \% \mathrm{Cl} 0.95-1.04$, $P$ for gender interaction, 0.096). Neighbourhood income deprivation was associated with victimisation in men and women with similar magnitudes of association.

\section{Conclusions}

Neighbourhood factors influencing safety, as well as individual characteristics including gender, may be relevant to understanding pathways to physical victimisation towards people with mental illness.

\section{Keywords}

Natural language processing; violence; neighbourhood characteristics; electronic health records; data linkage.

\section{Copyright and usage}

(c) The Author(s) 2020. Published by Cambridge University Press on behalf of The Royal college of Psychiatrists. This is an Open Access article, distributed under the terms of the creative Commons Attribution licence (http://creativecommons.org/ licenses/by/4.0/), which permits unrestricted re-use, distribution, and reproduction in any medium, provided the original work is properly cited.

\section{Background}

Physical violence is a common and preventable cause of morbidity and mortality in people with mental illness ${ }^{1}$ and has a negative impact on quality of life and treatment response. ${ }^{2}$ A 2016 systematic review of 30 studies found a strong association of severe mental illness with victimisation in both men and women, in comparison with the general population. ${ }^{3}$ Large register-based epidemiological studies in the USA, ${ }^{4}$ Sweden $^{5}$ and Denmark ${ }^{6}$ confirm the association between mental disorders and subsequent experience of violent crime.

\section{Victimisation}

The World Health Organization ecological framework emphasises neighbourhood and community context, alongside victim and perpetrator characteristics, in the occurrence of violence. Victimisation displays important gender differences; in the general population, men experience greater physical victimisation than women, whereas violence in domestic settings affects more women than men. ${ }^{8}$ In surveys of the general population, experiencing physical violence as a victim (physical victimisation) is associated with individual characteristics such as younger age, minority ethnicity, single marital status and use of drugs and alcohol, but also with neighbourhood deprivation, ${ }^{9}$ and residing in areas with greater population density. ${ }^{10}$ Risk of victimisation is influenced by the availability of settings where violence may more easily occur, the likelihood of interacting with a possible perpetrator and the local presence of risk factors for violence. The level of neighbourhood crime has therefore been evaluated as a risk factor for victimisation in the general population. ${ }^{11}$

\section{Neighbourhood characteristics}

Neighbourhood characteristics are also associated with mental illness. Neighbourhood deprivation is associated with occurrence of mental illness, including psychosis ${ }^{12}$ and depression. ${ }^{13}$ It has also been suggested that public mental health may be improved by nurturing neighbourhood social networks and local reserves of material resources, support, and trusting relationships accessible by people when they experience stress, adversity and disadvantage. ${ }^{14}$ A construct that captures these aspects is neighbourhood fragmentation, defined as the degree of social disorganisation, residential turnover and relationship breakdown in a neighbourhood. Fragmented neighbourhoods may display greater occurrence of severe mental illness, ${ }^{15,16}$ after accounting for individual characteristics. ${ }^{17}$

\section{Aims}

Identifying risk factors for physical victimisation in mental illness may provide avenues for developing effective interventions. 
However, there has been limited examination of neighbourhood characteristics as influences on physical victimisation in people with mental illness. Previous epidemiological investigations have typically used participant interviews, routine data and surveys to ascertain physical victimisation, with each method introducing possible differential under-ascertainment of all physical victimisation affecting the population. ${ }^{18}$ Physical victimisation identified by clinical services, for example during patient assessments and historytaking, could reflect incidents not collected through other sources, and strengthen evidence for interventions to improve patient safety. We examined neighbourhood effects on physical victimisation towards people using mental health services, testing genderspecific associations with neighbourhood characteristics within a multilevel conceptual framework.

\section{Method}

\section{Data source}

The study was carried out in accordance with the RECORD statement ${ }^{19}$ (see Supplementary Checklist, available at https://doi.org/ 10.1192/bjo.2020.52). We did a case-control study using two linked databases, one containing mental health records and another containing hospital admissions data. We used a natural language processing (NLP) algorithm, developed and evaluated for the purposes of this study to define 'cases' and controls.

Algorithm development and evaluation is described further in the Supplementary Methods. The first data source for this study was the South London and Maudsley (SLaM) Biomedical Research Centre Clinical Record Interactive Search (CRIS) system, comprising complete de-identified electronic health records from the comprehensive National Health Service (NHS) mental healthcare provider in South East London, offering services for residents of the London boroughs of Lambeth, Southwark, Lewisham, and Croydon (comprising a total population of around 1.2 million $^{20}$ ).

The register included data from clinical free text entered by clinicians, documents of clinical correspondence, structured fields for scales/questionnaires and sociodemographic data, since 2006 for all SLaM services. The CRIS database has been linked to the Hospital Episode Statistics (HES) Admitted Patient Care (APC) (HES-APC) data-set, a comprehensive record of all NHS hospital in-patient admissions in England since 2014. ${ }^{21}$ These linked data were used to provide information on use of in-patient medical care for victimisation. Alongside the NLP-derived definition of cases (described in the methodological supplement) we also inspected associations with a case definition based on HES admission for assault, drawn from linkage with the HES-APC data-set. The aim of using hospital admission cases was to check if associations with the NLP case definition were consistent in their magnitude/direction, when using a different case definition (incorporating HES data). See Fig. 1 for a flow diagram summarising the linked databases and flow of participants through the study.

\section{Consent and ethics statement}

All individual data was anonymous. Therefore, informed consent from participants was not sought. The authors assert that all procedures contributing to this work comply with the ethical standards of the relevant national and institutional committees on human experimentation and with the Helsinki Declaration of 1975, as revised in 2008. Ethical approval for CRIS was granted by the Oxford REC, reference 18/SC/0372.

\section{Identification of physical victimisation cases and controls}

The algorithm was applied to CRIS (15 May 2017; 284272 individual patient records). The algorithm generated a binary variable for each participant, for any clinical documentation of physical victimisation, occurring at any time in a person's lifetime (and recorded in clinical records from 2006). This variable was used to ascertain people with a very high probability of lifetime physical victimisation, who were defined as cases. For each case, ten controls, defined as individuals who were not identified with physical victimisation by the NLP algorithm, with referral dates falling within 1 year of the corresponding case, were also randomly sampled. This was in order to optimise power to detect possibly small associations. Linkage of CRIS with medical in-patient data from HES data for England and Wales had been previously established and is described elsewhere. ${ }^{20}$ We used this linkage to examine the case definition for our analyses. To identify admissions for victimisation, we used these linked data to identify the presence of at least one hospital admission involving ICD-10 codes ${ }^{22}$ for assault, which were: X85-99, Y00-Y04 and Y08-Y09. The diagnostic codes included in the HES definition of hospital admission for physical victimisation for this study are displayed in Supplementary Table 2.

\section{Neighbourhood characteristics}

Addresses at which cases and controls were residing at the time of referral to mental health services were used to derive information on neighbourhood characteristics. All neighbourhood characteristics were taken at the geographic level of the 2011 lower super output areas (LSOA), which are small geographic units enclosing an average of 1500 residents. Neighbourhood crime was measured using the Index for Multiple Deprivation crime domain for $2010 .^{23}$ Neighbourhood fragmentation was measured using the Congdon Index for neighbourhood fragmentation, ${ }^{24}$ a composite indicator based on 2011 data on population turnover, percentage of privately rented households, single person households and unmarried people. Neighbourhood socioeconomic status was measured using the income deprivation domain of the Index for Multiple Deprivation 2010. We also assessed the impact of including overall neighbourhood deprivation, rather than neighbourhood income deprivation, on estimates. Population density was measured using persons per hectare, based on census data from 2011. All neighbourhood characteristics data were positively scaled (i.e. higher scores indicating greater crime, fragmentation and income deprivation, respectively), and $z$-standardised for ease of interpretation of estimates, to reflect a mean of 0 and a s.d. of 1 . The measurement of other analysed variables is described in the methodological supplement.

\section{Analysis}

Analyses were carried out in Stata 14. We described counts, proportions, and $\chi^{2}$-tests of physical victimisation with age at referral (categorised for descriptive purposes into age groups 0-15, 16-24, 25-35, $36-50, \geq 51$ ), gender, ethnic group, marital status, primary diagnosis, the presence of comorbid drug or alcohol use disorders, and any record for hospital admission for physical victimisation in HES. Crude associations of physical victimisation with neighbourhood characteristics (neighbourhood fragmentation, neighbourhood crime, neighbourhood income deprivation, and population density) were described by comparing medians, means and $t$-tests. The correspondence of NLP-derived physical victimisation with hospital admission data was assessed by calculating the proportion of the case groups with at least one hospital admission for physical victimisation, and by reporting this proportion within strata of covariates included in this study, including neighbourhood characteristics (presented in Supplementary Table 3). 


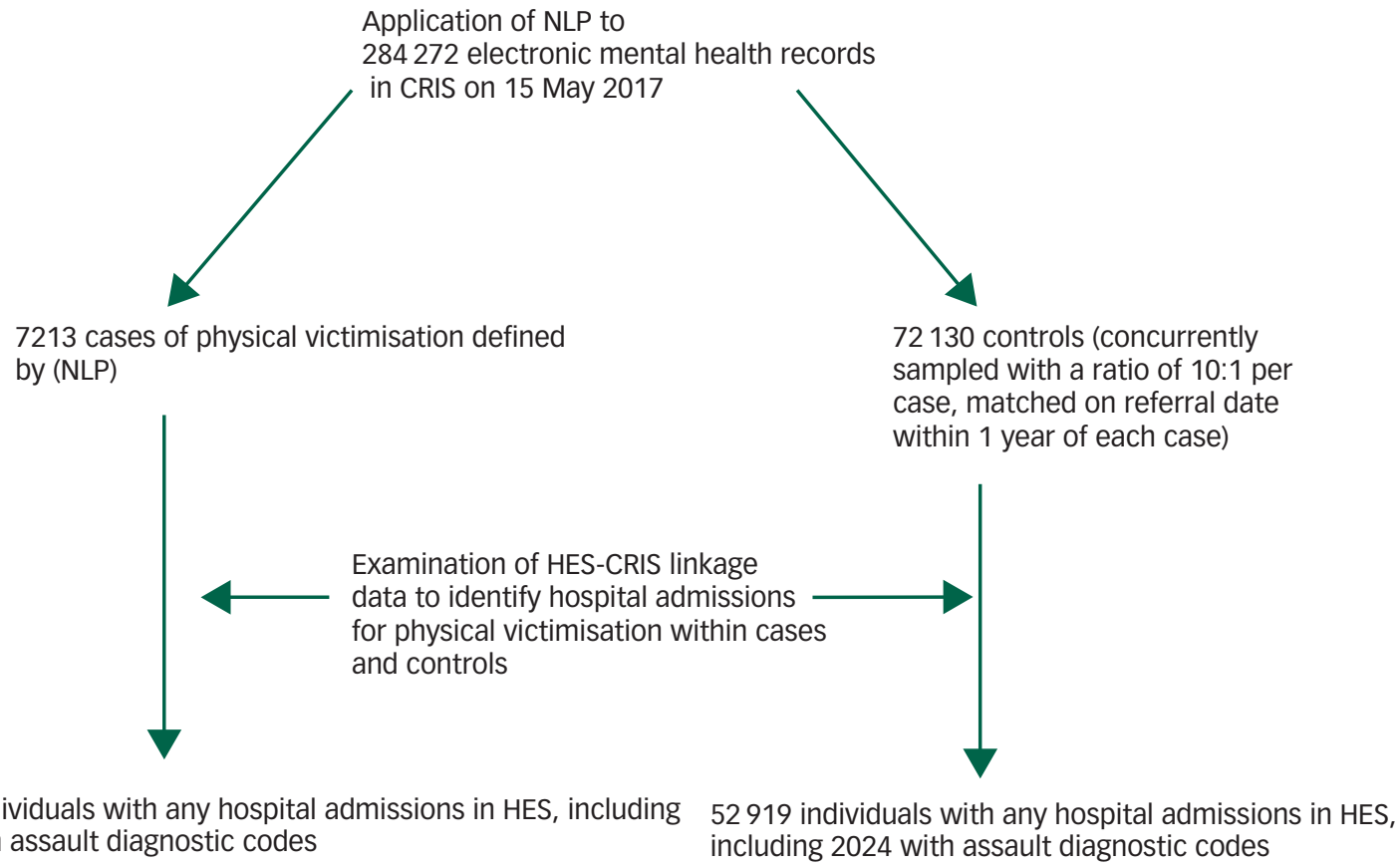

6298 individuals with any hospital admissions in HES, including 637 with assault diagnostic codes including 2024 with assault diagnostic codes

\section{Fig. 1 Flow diagram to demonstrate linked databases included in this study.}

NLP, natural language processing; HES, Hospital Episode Statistics; CRIS, Clinical Record Interactive Search.

We then modelled association between neighbourhood characteristics (neighbourhood fragmentation, neighbourhood crime, neighbourhood income deprivation and neighbourhood population density) and physical victimisation, based on the NLP algorithm. Because all neighbourhood characteristics were $z$-standardised (that is, set to have a mean of 0 and s.d. of 1 ), all logistic regression model coefficients for neighbourhood characteristics reflected the relative change in odds of physical victimisation for an increase of 1 s.d. in the neighbourhood characteristic. To evaluate collinearity affecting the stability and precision of model estimates, crude correlations among neighbourhood characteristics were evaluated using pairwise correlation coefficients and presented in a matrix (see Supplementary Table 4). Continuous variables were not entered in models together if the pairwise correlation between the two variables was greater than 0.7. All continuous covariates were assessed for goodness of fit as linear, quadratic and categorical indicator terms (in quintiles) using the Bayes Information Criteria.

In order to account for the clustering of neighbourhood characteristics within individuals residing in the same neighbourhoods, all models included a neighbourhood (LSOA)-level random effect, using the melogit command in Stata, and were estimated using robust standard errors. In primary analyses, age, gender, a multiplicative interaction term for gender, marital status and ethnic group were included in final models as forced covariates. Diagnostic group, and comorbid drug or alcohol use disorders, were evaluated for inclusion in final models, so as to maintain parsimony of the model. These covariates were included only if their inclusion changed the estimate by greater than $10 \%$ compared with the crude association. ${ }^{25}$

Having identified covariates for inclusion in the final model, adjusted estimates were reported by estimating random effects logistic regression models including (a) each neighbourhood characteristic without other covariates, (b) adding only individuallevel covariates, (c) by adding only the other neighbourhood covariates, and (d) including all variables in order to arrive at a fully adjusted estimate. All models employed linear combinations estimating gender-specific associations between neighbourhood characteristics and physical victimisation, and we reported model estimates associations for women, and post-estimation fitted estimates for the association in men. Finally, we estimated absolute risk differences for a difference in 1 s.d. from the mean, for neighbourhood characteristics by gender, based on final model estimates. Missing data on all variables included in final models were described by case-control status, and missing data proportions compared.

\section{Results}

\section{Descriptive results}

Based on a human-rated gold standard, the NLP algorithm had a positive predictive value of 0.92 and sensitivity of 0.98 for (clinically recorded) physical victimisation. We identified 7213 users of mental health services with a history of physical victimisation based on the NLP algorithm described, giving an overall prevalence of $2.5 \%$. Comparison of this group with 72130 concurrently sampled controls, without recorded physical victimisation, indicated association of physical victimisation with younger age, male gender, Black and mixed, ethnic group, and single and divorced marital status (all $P<$ 0.001 , see Table 1 ). Individuals with physical victimisation were most commonly diagnosed with psychotic disorders (20.4\%) and mood disorders (16.3\%). Based on HES-linkage, $8.8 \%$ of those identified as cases through NLP experienced at least one hospital admission for physical victimisation (Table 1).

Case status was associated with greater neighbourhood fragmentation, higher neighbourhood crime, and higher neighbourhood income deprivation, and greater population density, compared with controls (all $P<0.001$, Table 2). NLP-defined cases who also experienced hospital admission for physical victimisation were more commonly from younger age groups, men, of single marital status, diagnosed with comorbid alcohol and drug use disorders, and resided in neighbourhoods with lower neighbourhood income deprivation (Supplementary Table 3).

Pairwise correlations all suggested low or moderate correlation among neighbourhood fragmentation, neighbourhood crime, 
Table 1 Descriptive data on cases, with natural language processing-derived physical victimisation in health records, and controls, with column percentages for each covariate

\begin{tabular}{|c|c|c|c|c|c|}
\hline & $\begin{array}{l}\text { Control } \\
\text { group }\end{array}$ & $\begin{array}{l}\text { Case } \\
\text { group }\end{array}$ & Total & $\chi^{2}$ & $P$ \\
\hline \multicolumn{6}{|l|}{ Age, years } \\
\hline $0-15, n$ & 11261 & 1292 & 12554 & & \\
\hline $0-15, \%$ & 15.61 & 17.91 & 15.82 & & \\
\hline $16-24, n$ & 11780 & 1534 & 13314 & & \\
\hline $16-24, \%$ & 16.33 & 21.27 & 16.78 & & \\
\hline $25-35, n$ & 15769 & 1796 & 17565 & & \\
\hline $25-35, \%$ & 21.86 & 24.90 & 22.14 & & \\
\hline $36-50, n$ & 15840 & 1709 & 17549 & & \\
\hline $36-50, \%$ & 21.96 & 23.69 & 22.12 & & \\
\hline$\geq 51, n$ & 17410 & 878 & 18288 & & \\
\hline$\geq 51, \%$ & 24.14 & 12.17 & 23.05 & 560.60 & $<0.001$ \\
\hline Missing, $n$ & 70 & 3 & 73 & & \\
\hline Missing, \% & 0.10 & 0.04 & 0.09 & & \\
\hline \multicolumn{6}{|l|}{ Gender } \\
\hline Women, $n$ & 36510 & 3036 & 39546 & & \\
\hline Women, \% & 50.62 & 42.09 & 49.84 & & \\
\hline Men, $n$ & 35600 & 4177 & 39777 & & \\
\hline Men, \% & 49.36 & 57.91 & 50.13 & 191.30 & $<0.001$ \\
\hline Missing, $n$ & 20 & 0 & 20 & & \\
\hline Missing, \% & 0.03 & 0.00 & 0.03 & & \\
\hline \multicolumn{6}{|l|}{ Ethnic group } \\
\hline White, $n$ & 37156 & 3722 & 40878 & & \\
\hline White, \% & 51.51 & 51.60 & 51.52 & & \\
\hline Mixed, $n$ & 1751 & 337 & 2088 & & \\
\hline Mixed, \% & 2.43 & 4.67 & 2.63 & & \\
\hline Asian, $n$ & 2665 & 296 & 2961 & & \\
\hline Asian, \% & 3.69 & 4.10 & 3.73 & & \\
\hline Black, $n$ & 10483 & 2162 & 12645 & & \\
\hline Black, \% & 14.53 & 29.97 & 15.94 & & \\
\hline Other, $n$ & 4453 & 416 & 4869 & & \\
\hline Other, \% & 6.17 & 5.77 & 6.14 & 723.36 & $<0.001$ \\
\hline Missing, $n$ & 15622 & 280 & 15902 & & \\
\hline Missing, \% & 21.66 & 3.88 & 20.04 & & \\
\hline \multicolumn{6}{|l|}{ Marital status } \\
\hline Single, $n$ & 32499 & 4906 & 37405 & & \\
\hline Single, \% & 45.06 & 68.02 & 47.14 & & \\
\hline Married or cohabiting, $n$ & 10320 & 764 & 11084 & & \\
\hline Married or cohabiting, \% & 14.31 & 10.59 & 13.97 & & \\
\hline Divorced or separated, $n$ & 4143 & 571 & 4714 & & \\
\hline Divorced or separated, \% & 5.74 & 7.92 & 5.94 & & \\
\hline Widowed, $n$ & 3692 & 190 & 3882 & & \\
\hline Widowed, \% & 5.12 & 2.63 & 4.89 & 501.14 & $<0.001$ \\
\hline Missing, $n$ & 21476 & 782 & 22258 & & \\
\hline Missing, \% & 29.77 & 10.84 & 28.05 & & \\
\hline \multicolumn{6}{|l|}{ Primary ICD-10 diagnosis } \\
\hline F0-9: Organic mental disorders, $n$ & 4474 & 222 & 4696 & & \\
\hline F0-9: Organic mental disorders, \% & 6.20 & 3.08 & 5.92 & & \\
\hline F10-19: Mental and behavioural disorders due to psychoactive substance use, $n$ & 5647 & 523 & 6170 & & \\
\hline F10-19: Mental and behavioural disorders due to psychoactive substance use, \% & 7.83 & 7.25 & 7.78 & & \\
\hline F20-29: Schizophrenia, schizotypal and delusional disorders, $n$ & 3458 & 1472 & 4930 & & \\
\hline F20-29: Schizophrenia, schizotypal and delusional disorders, \% & 4.79 & 20.41 & 6.21 & & \\
\hline F30-39: Mood (affective) disorders, $n$ & 8647 & 1177 & 9824 & & \\
\hline F30-39: Mood (affective) disorders, \% & 11.99 & 16.32 & 12.38 & & \\
\hline F40-49: Neurotic, stress-related and somatoform disorders, $n$ & 6541 & 619 & 7160 & & \\
\hline F40-49: Neurotic, stress-related and somatoform disorders, \% & 9.07 & 8.58 & 9.02 & & \\
\hline $\begin{array}{l}\text { F50-59: Behavioural syndromes associated with physiological disturbances and physical } \\
\text { factors, } n\end{array}$ & 1944 & 65 & 2009 & & \\
\hline $\begin{array}{l}\text { F50-59: Behavioural syndromes associated with physiological disturbances and physical } \\
\text { factors, \% }\end{array}$ & 2.70 & 0.90 & 2.53 & & \\
\hline F60-69: Disorders of adult personality and behaviour, $n$ & 898 & 237 & 1135 & & \\
\hline F60-69: Disorders of adult personality and behaviour, \% & 1.24 & 3.29 & 1.43 & & \\
\hline F70-79: Learning disability, $n$ & 758 & 156 & 914 & & \\
\hline F70-79: Learning disability, \% & 1.05 & 2.16 & 1.15 & & \\
\hline F80-89: Disorders of psychological development, $n$ & 1361 & 149 & 1510 & & \\
\hline F80-89: Disorders of psychological development, \% & 1.89 & 2.07 & 1.90 & & \\
\hline $\begin{array}{l}\text { F90-98: Behavioural and emotional disorders with onset usually occurring in childhood and } \\
\text { adolescence, } n\end{array}$ & 3171 & 403 & 3574 & & \\
\hline $\begin{array}{l}\text { F90-98: Behavioural and emotional disorders with onset usually occurring in childhood and } \\
\text { adolescence, \% }\end{array}$ & 4.40 & 5.59 & 4.50 & & \\
\hline
\end{tabular}




\begin{tabular}{|c|c|c|c|c|c|}
\hline & $\begin{array}{l}\text { Control } \\
\text { group }\end{array}$ & $\begin{array}{l}\text { Case } \\
\text { group }\end{array}$ & Total & $\chi^{2}$ & $P$ \\
\hline F99: Unspecified mental disorder, $n$ & 10075 & 760 & 10835 & & \\
\hline F99: Unspecified mental disorder, \% & 13.97 & 10.54 & 13.66 & & \\
\hline No axis 1 diagnosis, $n$ & 3244 & 338 & 3582 & & \\
\hline No axis 1 diagnosis, \% & 4.50 & 4.69 & 4.51 & & \\
\hline G: Diseases of the nervous system, $n$ & 203 & 14 & 217 & & \\
\hline G: Diseases of the nervous system, $\%$ & 0.28 & 0.19 & 0.27 & & \\
\hline A-E, H-Q: Other illness codes, $n$ & 238 & 12 & 250 & & \\
\hline A-E, H-Q: Other illness codes, \% & 0.33 & 0.17 & 0.32 & & \\
\hline R: Symptoms, signs and abnormal clinical and laboratory findings, not elsewhere classified, $n$ & 401 & 11 & 412 & & \\
\hline R: Symptoms, signs and abnormal clinical and laboratory findings, not elsewhere classified, \% & 0.56 & 0.15 & 0.52 & & \\
\hline S-Y: Injury, poisoning and external causes, $n$ & 96 & 12 & 108 & & \\
\hline S-Y: Injury, poisoning and external causes, \% & 0.13 & 0.17 & 0.14 & & \\
\hline Z: Factors influencing health status and contact with health services, $n$ & 11868 & 850 & 12718 & $3 \times 10^{3}$ & $<0.001$ \\
\hline Z: Factors influencing health status and contact with health services, \% & 16.45 & 11.78 & 16.03 & & \\
\hline Missing, $n$ & 9106 & 193 & 9299 & & \\
\hline Missing, \% & 12.62 & 2.68 & 11.72 & & \\
\hline \multicolumn{6}{|l|}{ Any comorbid diagnosis of drug or alcohol use disorder } \\
\hline No, $n$ & 71665 & 7111 & 78766 & & \\
\hline No, \% & 99.35 & 98.59 & 99.27 & & \\
\hline Yes, $n$ & 475 & 102 & 577 & & \\
\hline Yes, \% & 0.66 & 1.41 & 0.73 & 51.85 & $<0.001$ \\
\hline \multicolumn{6}{|l|}{ HES assault admission } \\
\hline No, $n$ & 70151 & 6576 & 76727 & & \\
\hline No, $\%$ & 97.26 & 91.17 & 96.70 & & \\
\hline Yes, $n$ & 1979 & 637 & 2616 & & \\
\hline Yes, \% & 2.74 & 8.83 & 3.30 & 762.1659 & $<0.001$ \\
\hline Total, $n$ & 72130 & 7213 & 79343 & & \\
\hline
\end{tabular}

neighbourhood income deprivation and neighbourhood population density (see Supplementary Table 4). Associations of each covariate with physical victimisation did not vary when using a more restrictive outcome definition, based on the presence of both the NLP case definition and hospital admission for physical victimisation (see Supplementary Table 5).

\section{The associations of neighbourhood characteristics with physical victimisation}

Table 3 presents partially and fully adjusted model estimates for women and for men, all based on 44475 individuals with complete

\begin{tabular}{|c|c|c|c|}
\hline & $\begin{array}{l}\text { Control } \\
\text { group }\end{array}$ & $\begin{array}{l}\text { Case } \\
\text { group }\end{array}$ & $t$-test $P$ \\
\hline $\begin{array}{l}\text { Neighbourhood fragmentation, } \\
\text { mean (s.d.) }\end{array}$ & $0.00(1.00)$ & $0.08(0.91)$ & $<0.001$ \\
\hline $\begin{array}{l}\text { Neighbourhood fragmentation, } \\
\text { median (IQR) }\end{array}$ & $0.02(1.30)$ & $0.06(1.16)$ & \\
\hline Neighbourhood crime, mean (s.d.) & $0.27(0.81)$ & $0.41(0.74)$ & $<0.001$ \\
\hline $\begin{array}{l}\text { Neighbourhood crime, median } \\
\text { (IQR) }\end{array}$ & $0.36(0.99)$ & $0.47(0.85)$ & \\
\hline $\begin{array}{l}\text { Neighbourhood income } \\
\text { deprivation, mean (s.d.) }\end{array}$ & $0.09(0.89)$ & $0.29(0.82)$ & $<0.001$ \\
\hline $\begin{array}{l}\text { Neighbourhood income } \\
\text { deprivation, median (IQR) }\end{array}$ & $0.08(1.33)$ & $0.33(1.20)$ & \\
\hline $\begin{array}{l}\text { Neighbourhood population density, } \\
\text { mean (s.d.)) }\end{array}$ & $0.28(0.97)$ & $0.41(0.91)$ & $<0.001$ \\
\hline $\begin{array}{l}\text { Neighbourhood population density, } \\
\text { median (IQR) }\end{array}$ & $0.20(1.25)$ & $0.34(1.18)$ & \\
\hline
\end{tabular}

data on modelled variables, clustered in 2794 LSOA. For women, neighbourhood fragmentation was associated with $11 \%$ higher odds of physical victimisation, which attenuated to $5 \%$ (odds ratio $(\mathrm{OR})=1.05,95 \%$ CI 1.01-1.11) on adjustments. Neighbourhood crime was associated with $30 \%$ higher odds of physical victimisation in women, brought down to $7 \%$ after adjustments $(\mathrm{OR}=1.07,95 \%$ CI 1.01-1.14, see Table 4).

Before adjustments, greater neighbourhood income deprivation was associated with $29 \%$ higher odds of physical victimisation in women, which was attenuated to $14 \%$ after adjustment for individual and neighbourhood characteristics (OR $=1.14$, 95\% CI 1.08 2.21). Higher neighbourhood population density was associated with a $17 \%$ greater odds of victimisation in women, however, this was substantially attenuated on adjustment (fully adjusted $\mathrm{OR}=$ $0.99,95 \%$ CI 0.94, 1.04).

In men, there was a null association of neighbourhood fragmentation with physical victimisation after all adjustments $(\mathrm{OR}=1.00$, 95\% CI 0.95-1.04). There was a slightly higher point estimate for association of neighbourhood crime with physical victimisation in men $(\mathrm{OR}=1.13,95 \%$ CI 1.06-1.21). Neighbourhood income deprivation was associated with a $10 \%$ increase in the odds of victimisation in men after adjustments $(\mathrm{OR}=1.10$, 95\% CI $1.04-$ 1.16). The fully adjusted association of neighbourhood population density and victimisation in men was close to null $(\mathrm{OR}=0.97$, 95\% CI 0.92-1.01).

Our aims focused on associations between neighbourhood characteristics with victimisation in men and women. Therefore, we did not produce estimates pooled across men and women in the primary analysis but report these in Supplementary Table 6. Estimates were unchanged when we included neighbourhood deprivation, rather than neighbourhood income deprivation in final models. Absolute risk differences ranged from $-0.38 \%$ ( $95 \% \mathrm{CI}-0.09$ to 0.13 ) for neighbourhood 
Table 3 Model estimates for association of neighbourhood characteristics, based on 44475 records with complete data, clustered in 2794 neighbourhoods (lower super output areas)

\begin{tabular}{|c|c|c|c|c|c|}
\hline & Unadjusted $^{a}$ & $\begin{array}{l}\text { Individual- } \\
\text { adjusted }^{\text {b }}\end{array}$ & Area-adjusted ${ }^{c}$ & $\begin{array}{l}\text { Both individual- and area- } \\
\text { adjusted }^{\text {d }}\end{array}$ & $\begin{array}{c}\text { Absolute risk difference } \\
\%^{\mathrm{e}}\end{array}$ \\
\hline \multicolumn{6}{|l|}{ Women } \\
\hline $\begin{array}{l}\text { Neighbourhood } \\
\text { fragmentation }\end{array}$ & 1.11 (1.06 to 1.16$)$ & 1.06 (1.02 to 1.11$)$ & 1.07 (1.02 to 1.12 ) & 1.05 (1.01 to 1.11$)$ & 0.51 (0.02 to 1.00$)$ \\
\hline Neighbourhood crime & 1.30 (1.24 to 1.38$)$ & 1.19 (1.13 to 1.26$)$ & 1.13 (1.06 to 1.21$)$ & 1.07 (1.01 to 1.14$)$ & $0.59(-0.06$ to 1.23$)$ \\
\hline $\begin{array}{l}\text { Neighbourhood income } \\
\text { deprivation }\end{array}$ & 1.29 (1.24 to 1.34$)$ & 1.18 (1.13 to 1.24$)$ & 1.20 (1.14 to 1.27$)$ & 1.14 (1.08 to 1.21$)$ & 1.26 (0.72 to 1.81$)$ \\
\hline $\begin{array}{l}\text { Neighbourhood population } \\
\text { density }\end{array}$ & 1.17 (1.12 to 1.22$)$ & 1.08 (1.03 to 1.12$)$ & 1.05 (1.00 to 1.11$)$ & 0.99 (0.94 to 1.04$)$ & $-0.09(-0.56$ to 0.39$)$ \\
\hline \multicolumn{6}{|l|}{ Men } \\
\hline $\begin{array}{c}\text { Neighbourhood } \\
\text { fragmentation }\end{array}$ & 1.06 (1.01 to 1.11$)$ & 1.01 (0.97 to 1.05 ) & 1.03 (0.98 to 1.08$)$ & $1.00(0.95 \text { to } 1.04)^{f}$ & $-0.02(-0.51$ to 0.48$)$ \\
\hline Neighbourhood crime & 1.28 (1.21 to 1.34$)$ & 1.18 (1.12 to 1.24$)$ & 1.19 (1.11 to 1.28$)$ & $1.13(1.06 \text { to } 1.21)^{g}$ & 1.36 (0.67 to 2.04$)$ \\
\hline $\begin{array}{l}\text { Neighbourhood income } \\
\text { deprivation }\end{array}$ & $1.21(1.16$ to 1.26$)$ & 1.12 (1.08 to 1.17$)$ & 1.15 (1.09 to 1.21$)$ & $1.10(1.04 \text { to } 1.16)^{h}$ & 1.01 (0.44 to 1.59$)$ \\
\hline $\begin{array}{l}\text { Neighbourhood population } \\
\text { density }\end{array}$ & 1.10 (1.06 to 1.15$)$ & 1.03 (0.99 to 1.08 ) & 1.00 (0.95 to 1.05$)$ & $0.97(0.92 \text { to } 1.01)^{i}$ & $-0.38(-0.09$ to 0.13$)$ \\
\hline \multicolumn{6}{|c|}{$\begin{array}{l}\text { a. The intraclass correlation coefficient (\%) for the empty model, before inclusion of explanatory variables, was } 2.7(95 \% \mathrm{Cl} 2.0-3.8) \text {. } \\
\text { b. Adjusted for age, ethnic group, marital status, primary diagnosis and comorbid drug or alcohol use disorder. } \\
\text { c. Adjusted for the other neighbourhood characteristics. } \\
\text { d. Adjusted for age, ethnic group, marital status, primary diagnosis and comorbid drug or alcohol use disorder, and all other neighbourhood characteristics. } \\
\text { e. Based on the fully adjusted model, comparing absolute risks for a } 1 \text { s.d. increase in each neighbourhood characteristic. } \\
\text { f. P for gender by neighbourhood fragmentation interaction: } 0.096 \text {. } \\
\text { g. P for gender by neighbourhood crime interactionn: } 0.218 \text {. } \\
\text { h. P for gender by neighbourhood income deprivation interaction: } 0.257 \text {. } \\
\text { i. P for interaction between gender and neighbourhood population density: } 0.448 \text {. }\end{array}$} \\
\hline
\end{tabular}

population density in men, to $1.36 \%$ ( $95 \%$ CI 0.67 to 2.04 ), for neighbourhood crime in men.

\section{Covariate model estimates}

Among individual-level covariates, statistical associations with greater odds of physical victimisation were evident in final models for younger age, male gender, both mixed and Black ethnic groups (compared with the White reference group), divorced or separated marital status (compared with the single reference group), psychotic, mood, personality, and intellectual disability diagnostic groups (compared with the organic syndromes reference group), and the presence of a comorbid drug and alcohol use disorders (see Table 4). Married or cohabiting marital status was associated with a statistically lower odds of victimisation compared with single marital status.

\section{Missing data}

Complete data was included in models from 44475 individuals. Age and gender were missing in less than $1 \%$ of both the case and control groups (Supplementary Table 7). The case group had lower proportions of missing data than the controls for ethnic group, marital status and diagnosis. Missing data on neighbourhood fragmentation was between $11 \%$ and $15 \%$ for both the case and control groups, and between $5 \%$ and $7 \%$ for other neighbourhood characteristics. Crude associations between case-control status and neighbourhood within groups with missing data on each covariate were between 0.98 and 1.22 , consistent with final estimates in this study.

\section{Discussion}

\section{Summary of findings}

We present the first, to our knowledge, multilevel examination of neighbourhood associations with physical victimisation in people with mental illness, ascertained through NLP. We found evidence for association between neighbourhood fragmentation and physical victimisation among women, but not men. In contrast, neighbourhood crime and neighbourhood income deprivation, remained associated with physical victimisation in both women and men, after accounting for potential confounders, while crude associations of neighbourhood population density with victimisation were completely attenuated after covariate adjustment, in both men and women. In our final model, physical victimisation in people using mental health services was also associated with younger age at referral, male gender, divorced/separated marital status, Black ethnic group, mixed ethnic group, diagnosis of psychotic, mood, and personality disorders, and comorbid drug and alcohol use disorders. ${ }^{8}$

\section{Explanation of findings}

Our results suggest that although neighbourhood crime may influence physical victimisation in both men and women (after accounting for individual-level factors and neighbourhood income deprivation and population density), fragmentation is associated with physical victimisation in women, but not men, consistent with a small number of general population studies. ${ }^{26-29}$ Fragmented neighbourhoods are considered to offer more limited support structures at times of stress, need and privation, including access to third-sector support services. They may also offer fewer opportunities for meaningful activity, coping, safe physical activity and safe routines, which may all be necessary for maintaining personal safety. Neighbourhoods with greater levels of crime, antisocial behaviour, and rule breaking may also contain greater prevalence of perpetrators liable to commit crimes, including crimes towards people with vulnerabilities. It is possible that neighbourhood patterning of violent behaviour is one explanation for associations reported in this paper.

This violence could be more likely to occur outside the home, consistent with the gender differences observed in this study. On the other hand, violence against women with mental illness could be specifically driven by factors influencing the ability to access support, help, advocacy and resources in the wider community, partly reflected in the neighbourhood fragmentation variable investigated in this study. This is consistent with some literature on 
Table 4 All model estimates based on 44475 records with complete data, for the association, in the form of odds ratios (ORs with $95 \%$ Cls) of neighbourhood characteristics with physical victimisation

Neighbourhood characteristics

Neighbourhood fragmentation for women

Neighbourhood fragmentation for men ${ }^{\text {b }}$

Neighbourhood crime for women ${ }^{c}$

Neighbourhood crime for men

Neighbourhood income deprivation for women ${ }^{e}$

Neighbourhood income deprivation for men $^{\dagger}$

Neighbourhood population density for women ${ }^{8}$

Neighbourhood population density for men ${ }^{\mathrm{h}}$

Lower super output area-level random effect

Individual characteristics

Age $e^{i}$

Gender

Women

Men

Ethnic group

White

Mixed

Asian

Black

Other

Marital status

Single

Married or cohabiting

Divorced or separated

Widowed

Diagnostic group

F0-9: Organic mental disorders

F10-19: Mental and behavioural disorders due to psychoactive substance use

F20-29: Schizophrenia, schizotypal and delusional disorders

F30-39: Mood (affective) disorders

F40-49: Neurotic, stress-related and somatoform disorders

F50-59: Behavioural syndromes associated with physiological disturbances

F60-69: Disorders of adult personality and behaviour

F70-79: Learning disability

F80-89: Disorders of psychological development

F90-98: Behavioural and emotional disorders with onset

F99: Unspecified mental disorder

No Axis I diagnosis

$\mathrm{G}$ : Diseases of the nervous system

$A-E, H-Q$ : Other illness codes

R: Symptoms, signs and abnormal clinical and laboratory findings

S-Y: Injury, poisoning and external causes

Z: Factors influencing health status and contact with health services

Any comorbid diagnosis of drug or alcohol use disorders No

Yes

\subsection{5}

1.00

1.07

1.13

1.14

1.10

0.99

0.97

0.01

0.98

Reference

1.28

Ref

1.32

0.95

1.37

0.72

Reference

0.77

1.15

0.83

Reference

0.79

3.82

1.56

0.94

0.35

2.58

1.78

0.68

0.73

0.89

0.64

1.42

0.83

0.38

1.43

0.92

Reference

2.01

Lower 95\% Cl

Upper $95 \% \mathrm{Cl}$

a. A squared term for neighbourhood fragmentation was included based on goodness of fit in crude models for physical victimisation, and estimated at $0.99(95 \% \mathrm{Cl} 0.97-1.01)$

b. $P$ for gender by neighbourhood fragmentation interaction: 0.096 .

c. Neighbourhood crime was taken from the crime deprivation domain of the Index for Multiple Deprivation 2011.

d. $P$ for gender by neighbourhood crime interaction: 0.218 .

e. Neighbourhood income deprivation was taken from the income domain of the Index for Multiple Deprivation 2011.

f. $P$ for gender by neighbourhood income deprivation interaction: 0.257 .

g. Population density in people per square kilometre.

h. $P$ for interaction between gender and neighbourhood population density: 0.448 .

i. Age was included as a linear term in years, based on goodness of fit.

gender differences in neighbourhood associations with violence in the general population. In a study focusing specifically on dating violence, rather than any physical violence, Jain et $\mathrm{al}^{27}$ found that neighbourhood factors (concentrated poverty, perceived violence, collective efficacy) were more strongly associated with physical victimisation in men, than women. Cunradi and others ${ }^{30}$ examined the association of neighbourhood poverty with intimate partner violence, finding that statistical evidence remained after adjusting for income, marital status, number of children, educational attainment and socioeconomic status of both the victim and perpetrator. Although we were not able to distinguish between domestic and other types of violence in this study, it is possible that the observed neighbourhood differences between genders was related to neighbourhood factors being relevant to different types of physical victimisation.

In the general population, most violence occurs within the home, and in the context of intimate and family relationships. De Mooij et al examined the occurrence of victimisation in a Dutch out-patient sample of people with severe mental illness, which identified housemates as the most common perpetrators of victimisation $(21 \%)$, followed by neighbours, with a prevalence of $16 \% ;{ }^{31}$ although we had no information on the identity of perpetrators in this study, it is possible that neighbourhood patterns we observed were explained partly by an association between neighbourhood 
crime and the prevalence of perpetrators with propensity to be violent in each neighbourhood.

In the general population, routine activities theory has been proposed to explain observed neighbourhood variation in the occurrence of physical victimisation. This model suggests that physical victimisation occurs as a result of perpetrators and vulnerable would-be victims coming together in spaces that are poorly supervised, and where perpetrators may perceive there to be a lower risk of getting caught. ${ }^{32}$ The association we reported for neighbourhood fragmentation persisted after taking account of neighbourhood crime, suggesting that social networks and access to support could be relevant in protecting individuals from physical victimisation. More fragmented neighbourhoods may reflect weaker community structures allowing residents to live safely. It may be more difficult in fragmented neighbourhoods for people with existing vulnerabilities to access support that may protect against experiencing violence. It is also possible that other area characteristics, not measured in the current study, could account for the associations we observed. For example, research suggests geographic variation exists in gender norms, beliefs which condone domestic violence, ${ }^{33-35}$ and stigmatising attitudes towards people with mental illness; ${ }^{36,37}$ neighbourhood patterns in violence towards people with mental illness observed in this study could reflect regional differences in attitudes. Material deprivation in neighbourhoods is associated with the experience of violence, across a range of indicators, including intimate partner violence, ${ }^{38}$ homicide ${ }^{39}$ and use of hospital care for assault. ${ }^{40}$ Our findings confirm a relationship between neighbourhood deprivation and victimisation in people with mental disorder.

\section{Strengths and limitations}

Few studies to our knowledge have employed more than one method to identify or confirm physical victimisation in people with mental illness. The measurement of violence, and the possible role of gender in shaping measurement accuracy for different forms of violence, is complex ${ }^{41,42}$ and may call for a broad range of measurement approaches. We used NLP to identify cases, and investigated data from administrative linkage of mental health records data with acute medical admissions to confirm our results. In this study, we were able to demonstrate similarities between an NLP-derived physical victimisation measure based on clinical text, and diagnoses from acute medical in-patient admissions for the same population.

However, our focus on any clinical recording of physical victimisation in electronic health records did not distinguish between violence experienced in childhood, adulthood, or distinguish among different settings and perpetrators for violence (including identifying partners as perpetrators). Physical violence can be accompanied by other types of violence and abuse, including psychological coercion and control, financial abuse, and sexual exploitation/abuse, which were not directly assessed in this study, and may have a substantial impact on health outcomes. ${ }^{43}$ Residual confounding of our final estimates is likely, for example by family or household characteristics. The temporal relationship between physical victimisation and onset and occurrence of mental illness could not be evaluated. Given that our study focused on neighbourhood associations, the absence of information on where instances of physical victimisation took place is also a limitation. Individuals included in this study may have been residing in areas very different to those where instances of physical victimisation actually took place, which is also a limitation to the analysis.

Both NLP and hospital admission data on physical assault used in this study likely under-ascertained the occurrence of the outcome. We found a low prevalence of physical victimisation in comparison with other studies employing self-report. ${ }^{43,44}$ The NLP algorithm was developed to ascertain clearly worded instances of physical victimisation in the records, in order to minimise false positives, so this is likely to represent a subset of wider cases, accounting for the low overall prevalence of physical victimisation identified. Considering the primary findings, and given that the algorithm was developed using machine learning to ascertain clinically identified instance of physical victimisation, it is unlikely that this misclassification was driven by neighbourhood characteristics of interest in this study. Other possible explanations for a low prevalence of recorded physical victimisation in these routine data, could be low levels of enquiry for, and disclosure of, violence in clinical settings, which have been consistently observed.44 Perpetration of violence has also been linked to neighbourhood characteristics in previous research, however, information on whether individuals were perpetrators of violence was not available in this study.

In line with previous epidemiological literature on neighbourhood characteristics, we identified small effects, and absolute risk differences which ranged from $-0.38 \%$ to $1.36 \%$. The study was based on a population drawn from mental health services and is therefore only generalisable to that group - the study did not directly investigate pathways to physical victimisation in the general population. Cases were defined through NLP of electronic health records, and these cases could be non-representative of the mental health service user population as a whole, for example, individuals with extensive records, such as those with more severe illnesses necessitating greater recording, could have been over-represented among the case group than the control group. Conscious or unconscious bias affecting the recording of information by clinicians cannot be excluded as an explanation for the patterns shown. Few previous studies have examined clinical recordings of physical victimisation, and this may have allowed investigation of a different population to those captured in national crime data and general population surveys, given that mental illness is associated with non-participation and non-reporting of crimes in research studies. ${ }^{4}$ On the other hand, our findings may not be generalisable to people using mental health services experiencing physical victimisation but where this is not identified and clinically recorded by mental health professionals.

\section{Implications}

Physical violence towards people with mental illness is a fundamental health and social disparity. Where a person with mental illness lives may matter for their risk of experiencing physical violence. Strengthening social organisation and support in neighbourhoods could have an impact on the physical safety of people with mental illness, particularly women. As well as material deprivation, neighbourhood safety factors may be relevant for understanding physical violence towards people with mental illness. Public health strategies to improve the safety of community residents with mental illness could benefit from being gender sensitive. Incorporating information on physical victimisation from a number of measurement sources may be helpful for future research.

Vishal Bhavsar (DD, Section of Women's Mental Health, Institute of Psychiatry Psychology \& Neuroscience, King's College London, UK; Jyoti Sanyal, Clinical Informatics, BRC Nucleus, South London and Maudsley NHS Foundation Trust, UK; Rashmi Patel, Department of Psychosis Studies, Institute of Psychiatry, Psychology \& Neuroscience, King's College London, UK; Hitesh Shetty, Clinical Informatics, BRC Nucleus, South London and Maudsley NHS Foundation Trust, UK; Sumithra Velupillai, King's College London, UK; Robert Stewart (D), BRC Nucleus, South London and Maudsley NHS Foundation Trust, UK; Matthew Broadbent, Clinical Informatics, BRC Nucleus, South London and Maudsley NHS Foundation Trust, UK; James H. MacCabe (D), Department of Psychosis Studies, King's College London, UK; Jayati Das-Munshi (D), Department of Health Services and Population Research, King's College London, UK; Louise M. Howard (D), Section of Women's Mental Health, Institute of Psychiatry, Psychology \& Neuroscience, King's College London, UK

Correspondence: Vishal Bhavsar. Email: vishal.2.bhavsar@kcl.ac.uk

First received 20 Jan 2020, final revision 15 May 2020, accepted 2 Jun 2020 


\section{Supplementary material}

Supplementary material is available online at http://doi.org/10.1192/bjo.2020.52.

\section{Funding}

The study was conceived while $V . B$. was funded by a Wellcome Trust Clinical Research Training Fellowship $(101681 / \mathrm{Z} / 13 / \mathrm{Z})$. The research was carried out at the South London and Maudsley NHS Foundation Trust Biomedical Research Nucleus, which is funded by the National NHS Foundation Trust Biomedical Research Nucleus, which is funded by the National
Institute for Health Research. R.P. is funded by the Academy of Medical Sciences. S.V. was funded by the NIHR Biomedical Research Centre at the South London and Maudsley NHS Foundation Trust and King's College London. R.S. is part-funded by the NIHR Biomedical Research Centre at the South London and Maudsley NHS Foundation Trust and King's College London. RS is also part-funded by the Medical Research Council (MRC) Mental hata Pathfinder Award to King's Col Senior Investigator Award. J.M.D. is funded by the Health Foundation working together with the Academy of Medical Sciences, for a Clinician Scientist Fellowship and by the ESRC in relation to the SEP-MD study (ES/S002715/1) and part supported by the ESRC Centre for Society and Mental Health at King's College London (ESRC Reference: ES/S012567/1). The views expressed are those of the author(s) and not necessarily those of the NHS, the NIHR, the MRC, the Department of Health, the ESRC or King's College London.

\section{Data availability}

Researcher access to data used in this study is available on application to the SLaM BRC Case Register Oversight Committee.

\section{Author contributions}

Analyses were carried out by V.B., H.S. and J.S. The manuscript was finalised by L.M.H. and V.B. with substantial text contributions from J.S., R.P. S.V. and J.D.M. and further comments and significant input from all remaining co-authors. R.S. and M.B. also oversaw the planning and development of the SLaM BRC Case Register.

\section{Declaration of interest}

None.

\section{References}

1 Crump C, Sundquist K, Winkle MA, Sundquist J. Mental disorders and vulnerability to homicidal death: Swedish nationwide cohort study. BMJ 2013; 346 f557.

2 Goodman LA, Rosenberg SD, Mueser KT, Drake RE. Physical and sexual assault history in women with serious mental illness: prevalence, correlates, treatment, and future research directions. Schizophr Bull 1997; 23: 685-96.

3 Khalifeh H, Oram S, Osborn D, Howard LM, Johnson S. Recent physical and sexual violence against adults with severe mental illness: a systematic review and meta-analysis. Int Rev Psychiatry 2016; 28: 433-51.

4 Teplin LA, McClelland GM, Abram KM, Weiner DA. Crime victimization in adults with severe mental illness: comparison with the National Crime Victimization Survey. Arch Gen Psychiatry 2005; 62: 911-21.

5 Sariaslan A, Arseneault L, Larsson H, Lichtenstein P, Fazel S. Risk of subjection to violence and perpetration of violence in persons with psychiatric disorders in Sweden. JAMA Psychiatry 2020; 77: 359-67.

6 Dean K, Laursen TM, Pedersen CB, Webb RT, Mortensen PB, Agerbo E. Risk of being subjected to crime, including violent crime, after onset of mental illness: a Danish national registry study using police data. JAMA Psychiatry 2018; 75 689-96.

7 Krug EG, Mercy JA, Dahlberg LL, Zwi AB. The world report on violence and health. Lancet 2002; 360: 1083-8.

8 Brennan IR, Moore SC, Shepherd JP. Risk factors for violent victimisation and injury from six years of the British Crime Survey. Int Rev Vict 2010; 17: 209-29.

9 Sutherland A, Brunton-Smith I, Jackson J. Collective efficacy, deprivation and violence in London. Br J Criminol 2013; 53: 1050-74.

10 Nilsson A, Estrada F. Risky neighbourhood or individuals at risk? The significance of neighbourhood conditions for violent victimization in residential areas. J Scand Stud Criminol Crime Prev 2007; 8: 2-21.

11 Sampson RJ, Wooldredge JD. Linking the micro-and macro-level dimensions of lifestyle-routine activity and opportunity models of predatory victimization. $J$ Quant Criminol 1987; 3: 371-93.

12 Bhavsar V, Boydell J, Murray R, Power P. Identifying aspects of neighbourhood deprivation associated with increased incidence of schizophrenia. Schizophr Res 2014; 156: 115-21.
13 Fone D, White J, Farewell D, Kelly M, John G, Lloyd K, et al.. Effect of neighbourhood deprivation and social cohesion on mental health inequality: a multilevel population-based longitudinal study. Psychol Med 2014; 44: 2449-60.

14 Fagg J, Curtis S, Stansfeld SA, Cattell V, Tupuola A-M, Arephin M. Area social fragmentation, social support for individuals and psychosocial health in young adults: evidence from a national survey in England. Soc Sci Med 2008; 66: $242-54$.

15 Zammit S, Lewis G, Rasbash J, Dalman C, Gustafsson JE, Allebeck P. Individuals, schools, and neighborhood: a multilevel longitudinal study of variation in incidence of psychotic disorders. Arch Gen Psychiatry 2010; 67: 914-22.

16 Allardyce J, Gilmour H, Atkinson J, Rapson T, Bishop J, Mccreadie RG. Social fragmentation, deprivation and urbanicity: relation to first-admission rates for psychoses. Br J Psychiatry 2005; 187: 401-6.

17 Piantadosi S, Byar DP, Green SB. The ecological fallacy. Am J Epidemiol 1988; 127: 893-904.

18 Walby S, Towers J, Balderston S, Corradi C, Francis B, Heiskanen M, et al. The Concept and Measurement of Violence Against Women and Men. Policy Press, 2017.

19 Benchimol El, Smeeth L, Guttmann A, Harron K, Moher D, Petersen I, et al. The reporting of studies conducted using observational routinely-collected health data (RECORD) statement. PLoS Med 2015; 12: e1001885.

20 Perera G, Broadbent M, Callard F, Chang C-K, Downs J, Dutta R, et al. Cohort profile of the South London and Maudsley NHS Foundation Trust Biomedical Research Centre (SLaM BRC) case register: current status and recent enhancement of an electronic mental health record-derived data resource. BMJ Open 2016; 6: e008721.

21 Wijlaars L, Herbert A, Zylbersztejn A, Hardelid P, Cromwell D. Data resource profile: hospital episode statistics admitted patient care (HES APC). Int $J$ Epidemiol 2017; 46: 1093-i.

22 World Health Organization. The ICD-10 Classification of Mental and Behavioural Disorders: Diagnostic Criteria for Research (Vol. 2). WHO, 1993.

23 Office of the Deputy Prime Minister. Indices of Deprivation 2004. Office of the Deputy Prime Minister, 2004

24 Congdon P. Suicide and parasuicide in London: a small-area study. Urban Stud 1996; 33: 137-58.

25 Greenland S, Daniel R, Pearce N. Outcome modelling strategies in epidemiology: traditional methods and basic alternatives. Int J Epidemiol 2016; 45: 565-75.

26 Browning CR. The span of collective efficacy: extending social disorganization theory to partner violence. J Marriage Fam 2002; 64: 833-50.

27 Jain S, Buka SL, Subramanian S, Molnar BE. Neighborhood predictors of dating violence victimization and perpetration in young adulthood: a multilevel study. Am J Public Health 2010; 100: 1737-44.

28 Miller J, White NA. Gender and adolescent relationship violence: a contextual examination. Criminology 2003; 41: 1207-48.

29 Katz J, Kuffel SW, Coblentz A. Are there gender differences in sustaining dating violence? An examination of frequency, severity, and relationship satisfaction. J Fam Violence 2002: 17: 247-71.

30 Cunradi CB, Caetano R, Clark C, Schafer J. Neighborhood poverty as a predictor of intimate partner violence among White, Black, and Hispanic couples in the United States: a multilevel analysis. Ann Epidemiol 2000; 10: 297-308.

31 de Mooij LD, Kikkert M, Lommerse NM, Peen J, Meijwaard SC, Theunissen J, et al. Victimisation in adults with severe mental illness: prevalence and risk factors. Br J Psychiatry 2015; 207: 515-22.

32 Cohen $L E$, Felson $M$. Social change and crime rate trends: a routine activity approach. Am Sociol Rev 1979; 44: 588-608.

33 Antai D. Traumatic physical health consequences of intimate partner violence against women: what is the role of community-level factors? BMC Women's Health 2011; 11: 56

34 Mulawa MI, Reyes HLM, Foshee VA, Halpern CT, Martin SL, Kajula L, et al. Associations between peer network gender norms and the perpetration of intimate partner violence among urban Tanzanian men: a multilevel analysis. Prev Sci 2018; 19: 427-36.

35 Herrero J, Rodríguez FJ, Torres A. Acceptability of partner violence in 51 societies: the role of sexism and attitudes toward violence in social relationships. Violence Against Women 2017; 23: 351-67.

36 Bhavsar V, Schofield P, Das-Munshi J, Henderson C. Regional differences in mental health stigma - analysis of nationally representative data from the Health Survey for England, 2014. PLOS One 2019; 14: e0210834.

37 Gonzales L, Yanos PT, Stefancic A, Alexander MJ, Harney-Delehanty B. The role of neighborhood factors and community stigma in predicting community participation among persons with psychiatric disabilities. Psychiatr Serv 2018; 69: 76-83. 
38 Yakubovich AR, Heron J, Feder G, Fraser A, Humphreys DK. Long-term exposure to neighborhood deprivation and intimate partner violence among women: a UK birth cohort study. Epidemiology 2020; 31: 272-81.

39 Shaw M, Tunstall H, Dorling D. Increasing inequalities in risk of murder in Britain trends in the demographic and spatial distribution of murder, 1981-2000. Health Place 2005; 11: 45-54.

40 Bellis MA, Hughes K, Anderson Z, Tocque K, Hughes S. Contribution of violence to health inequalities in England: demographics and trends in emergency hospital admissions for assault. J Epidemiol Community Health 2008; 62: 1064-71.

41 Walby S, Towers J, Francis B. Is violent crime increasing or decreasing? A new methodology to measure repeat attacks making visible the significance of gender and domestic relations. Br J Criminol 2015; 56: 1203-34.

42 Schneider AL. Methodological problems in victim surveys and their implications for research in victimology. J Crim L Criminol 1981; 72: 818
43 Khalifeh H, Dean K. Gender and violence against people with severe mental illness. Int Rev Psychiatry 2010; 22: 535-46.

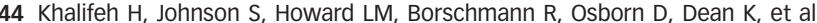
Violent and non-violent crime against adults with severe mental illness. $\mathrm{Br} J$ Psychiatry 2015; 206: 275-82.

45 Maniglio R. Severe mental illness and criminal victimization: a systematic review. Acta Psychiatr Scand 2009; 119: 180-91.

46 Trevillion K, Hughes B, Feder G, Borschmann R, Oram S, Howard LM. Disclosure of domestic violence in mental health settings: a qualitative meta-synthesis. Int Rev Psychiatry 2014; 26: 430-44. 